\title{
MATRIX METALLOPROTEINASE 9 (MMP-9) IS DIFFERENTLY EXPRESSED IN CUTANEOUS LICHEN PLANUS AND LICHEN SCLEROSUS
}

\author{
ILZE LEGUSA ${ }^{1}$, VALERIE GROMA ${ }^{2}$ \\ ${ }^{1}$ Clinical Centre for Skin and Sexually Transmitted Diseases, Riga, Latvia \\ ${ }^{2}$ Laboratory of Electron Microscopy, Institute of Anatomy and \\ Anthropology, Riga Stradiňs University, Riga, Latvia
}

\begin{abstract}
Lichen planus is a mucocutaneous inflammatory disease of unknown etiology. Hyperkeratosis, focal hypergranulosis, damage to the basal cell layer, and bandlike infiltrate are hallmarks of LP skin. Lichen sclerosus is a lymphocytemediated dermatosis that has a predilection for the genital skin in both sexes. Both pathologies are sharing a number of common characteristics. Previous studies have reported on involvement of matrix metalloproteinases and their capability of digesting extracellular matrix and basement membrane components. We report on the clinical examination of 11 lichen planus patients and 5 lichen sclerosus patients and the morphological evaluation of their skin biopsy samples. Clinical and routine light microscopy findings correlate with the literature data. By contrast, the expression of MMP-9 was greatly varying among clinical types of cutaneous lichen ruber planus. There was similarity in the MMP-9 expression observed between lichen planus pemphigoides and lichen sclerosus.
\end{abstract}

Key words: lichen planus, lichen sclerosus, matrix metalloproteinase 9 (MMP-9).

\section{INTRODUCTON}

Lichen planus (LP) is a mucocutaneous inflammatory disease of unknown etiology [1]. LP shares common chracteristics with the lupus erythematosus, benign lichenoid keratosis, keratosis lichenoides chronica, lichen notidus, and 
lichen sclerosus (LS) lesions. These pathologies are characterized by the damage of epidermis appearing mostly in the basal cell layer, and dermal infiltration. Lichen sclerosus is a lymphocyte-mediated dermatosis that has a predilection for the genital skin in both sexes. LP and LS are usually diagnosed clinically [2]. LP is characterized by flat-topped, pink to violaceous, shiny, pruritic polygonal papules that may coalesce into plaques. The typical clinical picture of LS is characterized by whitish, ivory or porcelain-white, sharply demarcated, pruritic, individual papules that may become confluent and form plaques [3]. The aetiology of both diseases is uncertain [4]. The histologic examination of skin or mucosal biopsy is helpful in the cases where there is some clinical doubt about the diagnosis and in the documentation of atypical features. The classical histological features of LS include a thinned epidermis with hyperkeratosis, a wide band of homogenized collagen below the dermoepidermal junction and a lymphocytic infiltrate beneath the homogenized area [5]. Histologically, LP is characterized by basal layer vacuolization, acanthosis, hypergranulosis, and a lymphocytic infiltrate mostly localized at the dermalepidermal interface [6]. The lesions usually involve skin, the oral mucous membranes, and genitalia. Direct immunofluorescence studies may be helpful in the disease differentiation for the cases with no specific clinical or histologic characteristics, or with ambiguous features of other diseases [7]. The matrix metalloproteinases (MMPs) are a large family of zinc-dependent endopeptidases, which are capable of digesting extracellular matrix and basement membrane components. These enzymes can be produced by several types of cells in skin such as fibroblasts, keratinocytes, macrophages, lymphocytes, endothelial cells, mast cells, and eosinophils [8]. The MMPs are involved in many processes including cell proliferation, differentiation, migration, apoptosis, and angiogenesis. Previous studies [9] have shown that dermal inflammatory cells are strongly expressing MMP-9. The elucidation of the role of MMPs in pathogenesis of chronic dermatosis, in general, and LP, in particular, is far from definitive. The comparative evaluation of the clinical and pathomorphological features of LP and LS including the peculiarities related to the expression of MMP-9 in LS and various types of LP appears under the scope of this paper.

\section{MATERIALS AND METHODS}

Our target patients were at least 18 years old, suffering from LP or LS for at least 4 weeks, having visible characteristic LP or LS eruptions in typical localization sites. We selected 11 patients ( 7 female and 4 male patients) with the 
histologically confirmed diagnosis of LP and 5 patients (all the patients were females) with the histologically confirmed diagnosis of LS. All the patients were admitted and treated at the Clinical Centre for Skin and Sexually Transmitted Diseases, Riga, Latvia between February 2010 and February 2012. Punch biopsy tissues archived at the above mentioned Centre were examined using light microscopy. At the time of biopsy all the patients were off any topical or systemic LP or LS medication. Study procedures were conducted in accordance with the rules of the Ethical Committee.

Histological sections of 4-5 $\mu \mathrm{m}$ were cut from formalin-fixed, paraffinembedded punch biopsy tissues and mounted on slides. Consecutive sections were used as negative controls of the immunohistochemical reactions and for hematoxylin and eosin $(\mathrm{H} \& \mathrm{E})$ staining to confirm the diagnosis. For immunochemistry paraffin sections were dewaxed and transferred to a methanol/0.3\% hydrogen peroxide solution in order to abolish endogenous peroxidase activity. After quenching of endogenous peroxidase activity sections were washed three times in double distilled water. Heat-induced antigen retrieval was accomplished with the sections placed in $10 \mathrm{mM}$ citrate buffer for 30 minutes in a vapor lock. After antigen retrieval, specimens were allowed to cool for 20 minutes. Nonspecific binding was blocked with $1 \%$ bovine serum albumin $/ 5 \%$ normal goat serum in phosphate buffered saline. Thereafter, sections were incubated at $4^{\circ} \mathrm{C}$ overnight with the primary antibody(anti-MMP-9) at a dilution 1:50, and next day, with secondary biotinylated antibody (1:500 dilution) for $30 \mathrm{~min}$ and streptavidin-biotin-peroxidase preformed complex (1:250 dilution) for $30 \mathrm{~min}$. The immunological reaction was developed with 3, 3'-diaminobenzidine tetrahydrochloride $(50 \mathrm{mg}$ in $100 \mathrm{ml}$ of PBS with $0.03 \% \mathrm{v} / \mathrm{v}$ hydrogen peroxide). Sections were counterstained with Harrys haematoxylin and mounted in Kaiser's glycerol gelatin. The intensity of immunostaining was graded semiquantitatively using the following scale: $(0)$ - negative expression, $(+)$ - a weak expression of MMP-9, $(++)$ - a moderate expression of MMP-9 and $(+++)-$ an intense MMP-9 expression.

\section{RESULTS}

Small, flat-topped, shiny, polygonal, violaceous papules were limited to the flexor surfaces of the forearms, the legs, the back of the trunk and submammary folds. Histologically, the hypertrophic variant of LP, LP pemphigoides and follicular form of lichen planus were confirmed. Hypertrophic (three cases) appeared to be a common variant which consisted of thickened plaques and 
showed hyperkeratosis, focal hypergranulosis, basal cell layer degeneration, and a band-like subepidermal infiltration (Figure 1). LP pemphigoides (five cases) showed bullae arising from papules and normal appearing skin. There were a focus of epidermal thickening with liquefaction degeneration of the basal layer, a lichenoid inflammatory infiltrate, and, sometimes, fibrin deposition (Figure 2). Lichen follicularis (three cases) revealed dilated hair follicles containing keratotic plugs (Figure 3). There were rather dense chronic inflammatory infiltrates in the close vicinity to the hair follicles, and band-like infiltrates beneath the epidermis. In LS were observed hyperkeratosis with follicular plugging, the atrophy of the stratum malpighii with the hydropic degeneration of basal cells, lymphedema and the homogenization of the collagen in the upper dermis, an inflammatory infiltrate in the mid-dermis, and the homogenization and swelling of the collagen bundles in the lower dermis (Figure 4).

MMP-9 expression was observed to be greatly varying among the cases enrolled in this study (Table 1). In a hypertrophic variant moderate to intense expression of metalloproteinase was revealed within the basal and suprabasal location of the stratum spinosum (Figure 5). A diffuse and strong MMP-9 immunostaining throughout all epidermal layers was noticed in the follicular variant of LP (Figure 6). The first two types of LP demonstrated diffuse and intense MMP-9 immunostaining in dermal lymphohistiocytic infiltrate, hair follicle and dermal sweat glands. By contrast, LP pemphigoides (Figure 7) demonstrated a weak expression of MMP-9 to the basal layer of epidermis whereas intense expression in dermal lymphohistiocytic infiltrate. LS demonstrated mild epidermal anti-MMP-9 decoration, which became moderate in dermal lymphohistiocytic infiltrate, and intense in sweat glands (Figure 8). In LS inflammatory infiltrates occupied mid-dermis region comparing with subepidermal characteristic for LP. Moreover, LS showed anti-MMP-9 decoration related to collagen location. 


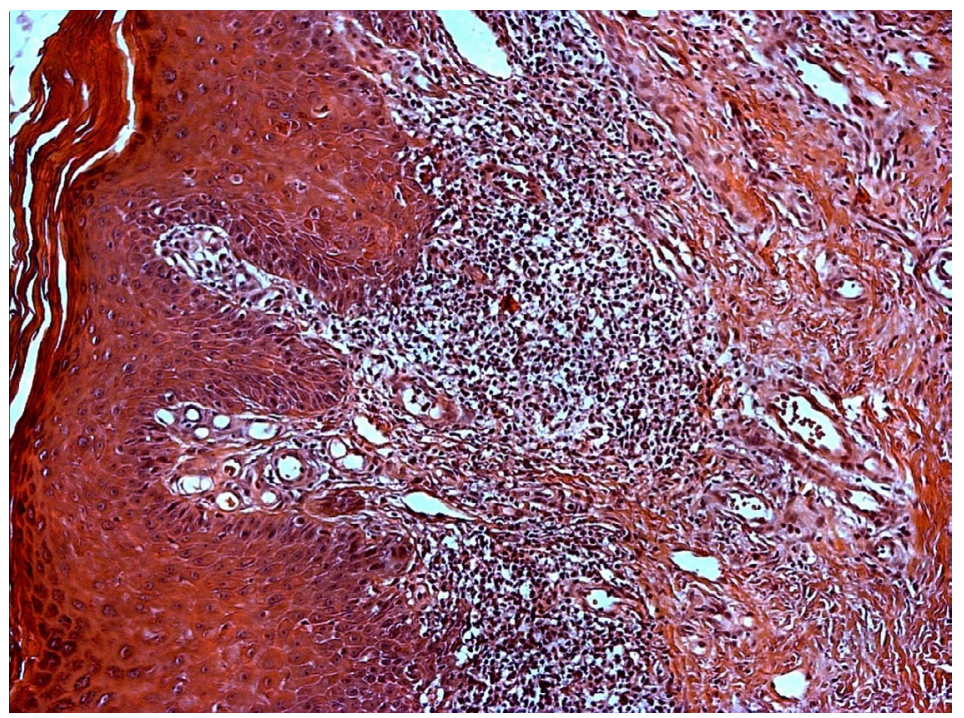

Figure 1. Hypertrophic LP with hyperkeratosis, hypergranulosis, saw-tooth profile of rete ridges, the degeneration of the basal cell layer, and compact band-like infiltrate below the epithelium. H\&E, original magnification $\times 100$.

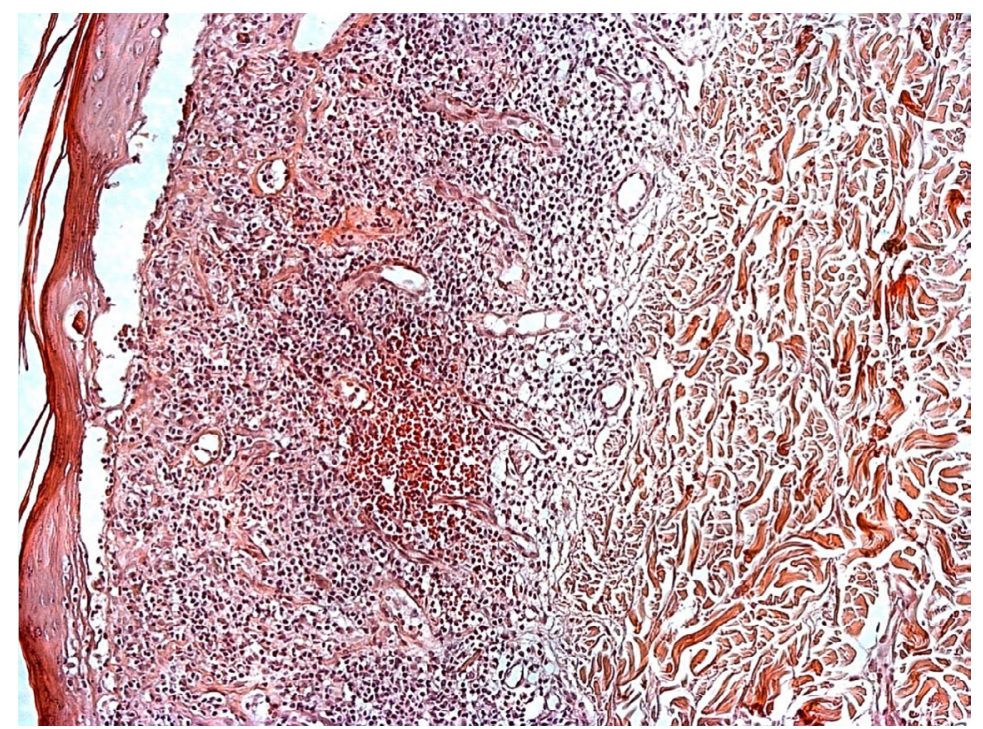

Figure 2. LP pemphigoides showing the separation of the epidermis from the dermis. A subepidermal bulla overlying a perivascular infiltrate, characteristic of LP pemphigoides. The liquefaction degeneration of the basement membrane. H\&E, original magnification $x$ 100. 


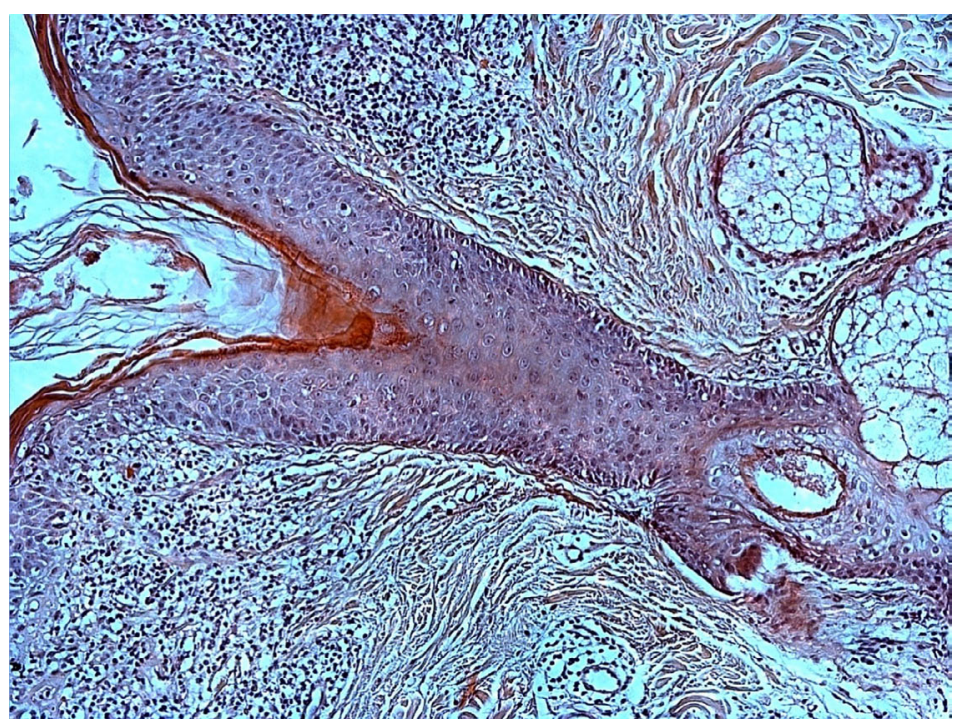

Figure 3. Follicular LP with the basal cell degeneration and subepidermal infiltrate. H\&E, original magnification $\times 100$.

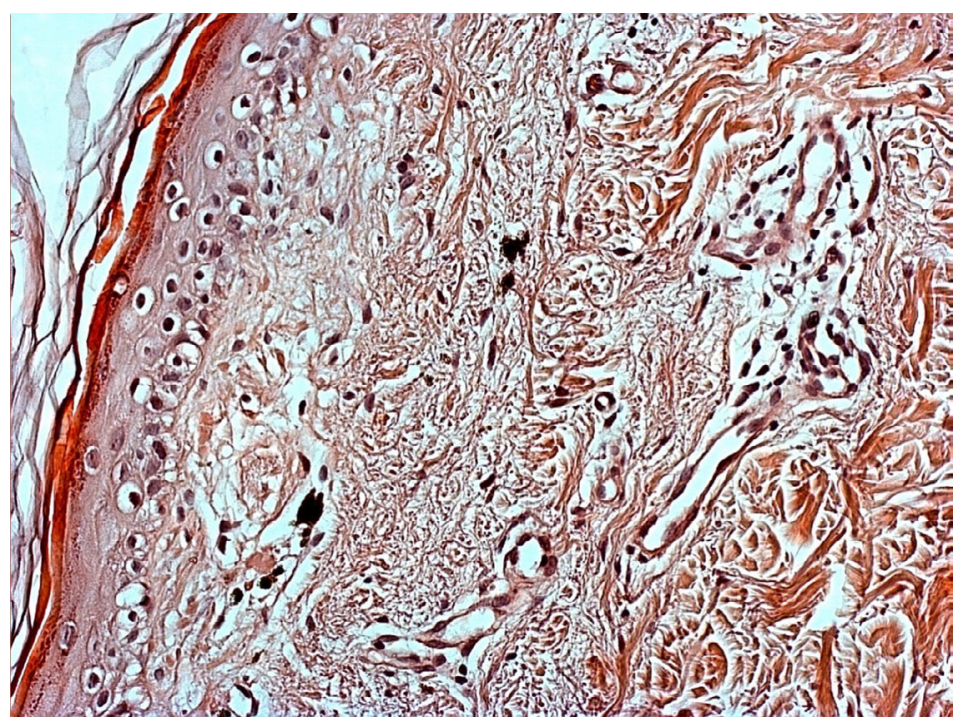

Figure 4. LS revealing hyperkeratosis and hypergranulosis, the atrophy of the stratum malpighii with the hydropic degeneration of basal cells, lymphedema and the homogenization of the collagen in the upper dermis, as well as a certain number of pigment cells. H\&E, original magnification $\times 200$. 
Table 1. Expression of MMP-9 in various clinical types of cutaneous LP and LS.

\begin{tabular}{|c|c|c|c|c|c|c|c|c|c|c|}
\hline \multicolumn{2}{|c|}{ Disease } & 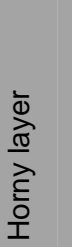 & 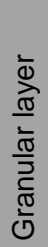 & 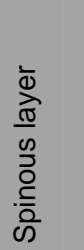 & 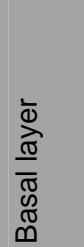 & 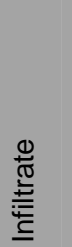 & 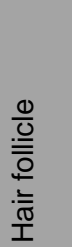 & 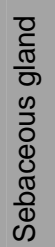 & 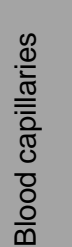 & 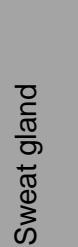 \\
\hline \multirow{3}{*}{$\begin{array}{l}\frac{0}{2} \\
\frac{\sigma}{\sigma} \\
\frac{\pi}{2} \\
\frac{\bar{d}}{d} \\
\frac{\bar{U}}{U}\end{array}$} & $\begin{array}{l}\text { Hypertrophic lichen } \\
\text { planus }\end{array}$ & $0 /+$ & 0 & $\begin{array}{l}++l \\
+++\end{array}$ & $\begin{array}{l}++l \\
+++\end{array}$ & +++ & +++ & 0 & ++ & +++ \\
\hline & $\begin{array}{l}\text { Follicular lichen } \\
\text { planus }\end{array}$ & 0 & 0 & $\begin{array}{l}++1 \\
+++\end{array}$ & $\begin{array}{l}++l \\
+++\end{array}$ & +++ & +++ & 0 & + & +++ \\
\hline & $\begin{array}{l}\text { Lichen planus } \\
\text { pemphigoides }\end{array}$ & 0 & 0 & $0 /+$ & + & +++ & ++ & 0 & $0 /+$ & $+/++$ \\
\hline \multicolumn{2}{|c|}{ Lichen sclerosus } & 0 & 0 & $0 /+$ & $+/++$ & +++ & $0 /+$ & 0 & $0 /+$ & +++ \\
\hline
\end{tabular}

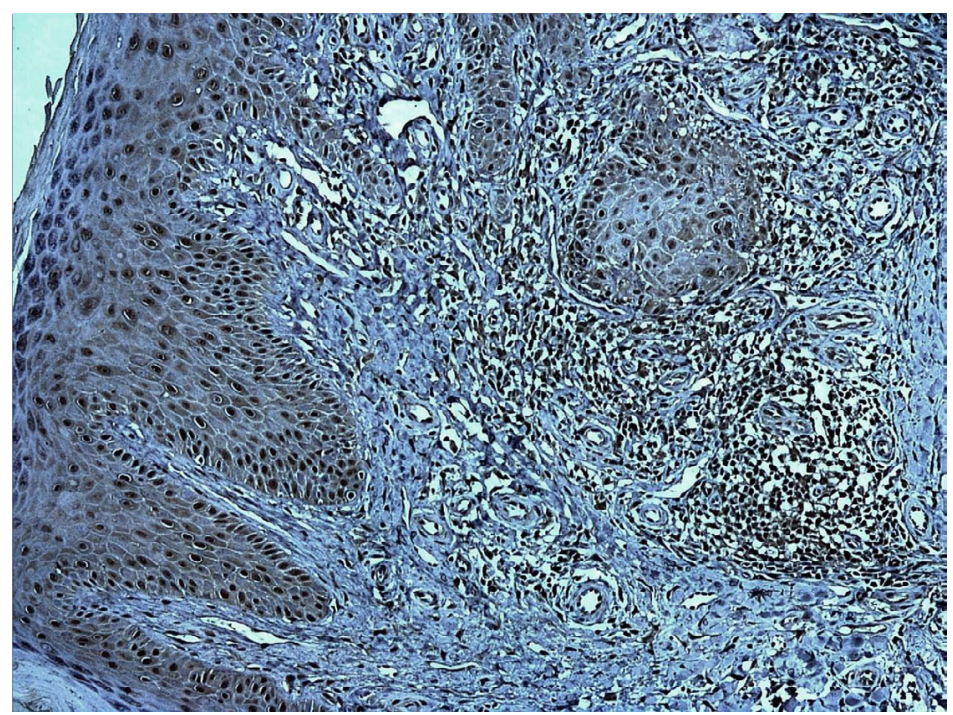

Figure 5. Expression of MMP-9 in hypertrophic LP appearing through the malpighii layer, dermal infiltrates, vascular beds, and sweat glands; original magnification $\times 100$. 


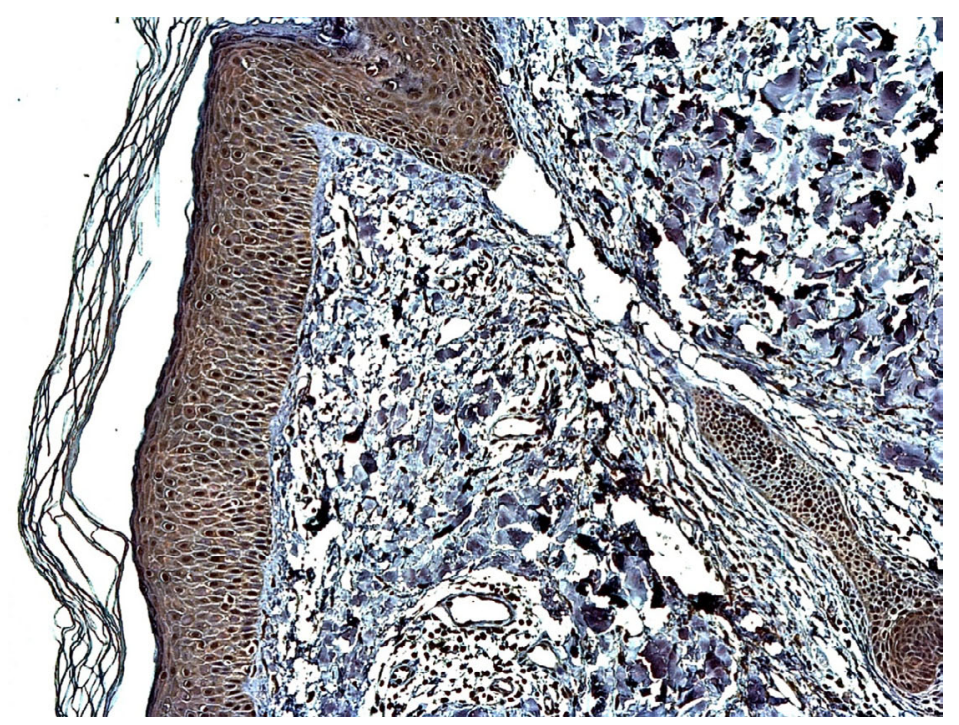

Figure 6. Epidermis and hair follicles appearing to be heavily decorated with the antiMMP-9 antibody in the follicular variant of LP, original magnification $\times 100$.

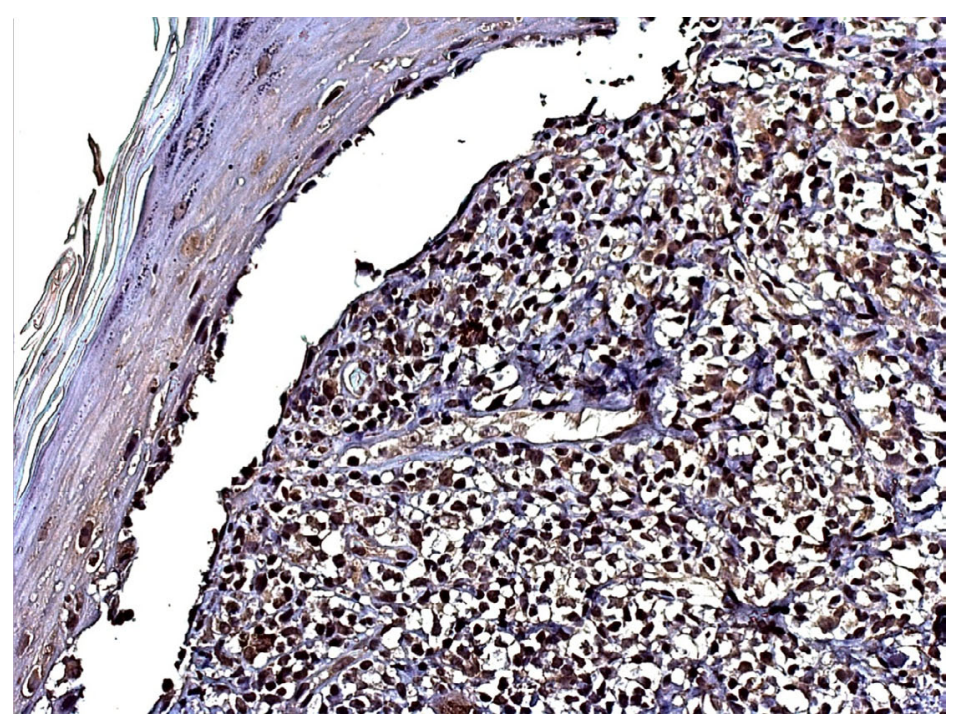

Figure 7. Mild epidermal and strong expression of MMP-9 in the inflammatory infiltrate demonstrated in LP, original magnification $\times 200$. 


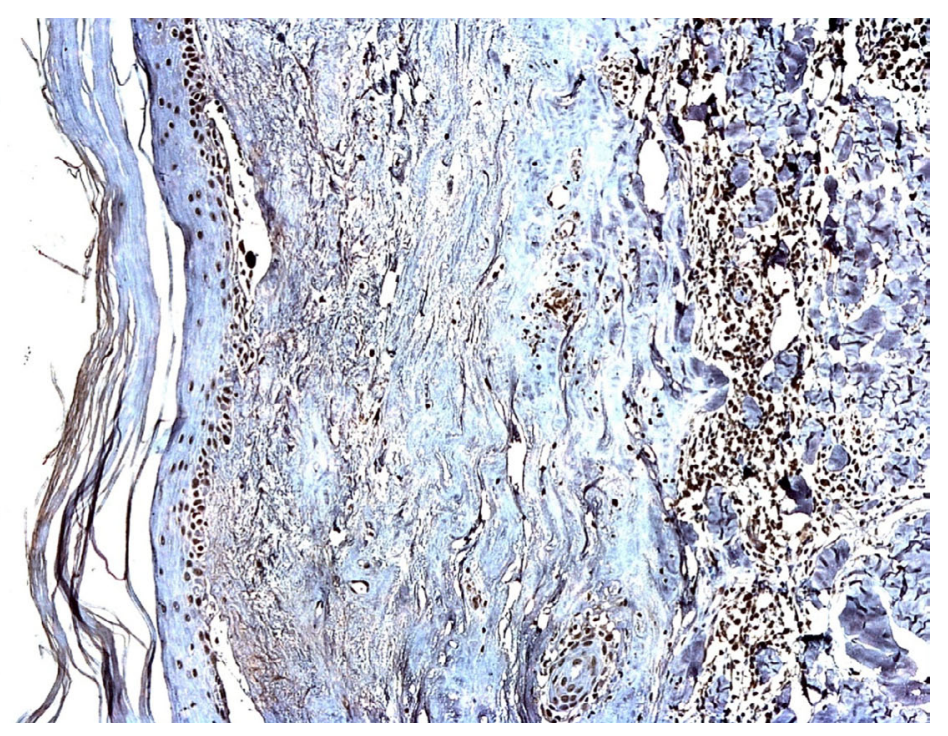

Figure 8. Mild epidermal and strong expression of MMP-9 in the inflammatory infiltrate localized in the mid-dermisin case of LS, original magnification $\times 100$.

\section{DISCUSSION}

We performed a complex clinical and pathomorphological study on the skin punch biopsy tissue with the confirmed LP diagnosis, and compared them with LS biopsy tissue samples. We found that an addition of immunohistochemistry data was more relevant and instructive as compared with solely used methods and examinations.

Our evidence regarding the contribution of MMPs to the events underlying these cutaneous pathologies is important for further understanding of intimate pathways of pathogenesis of LP.

We demonstrated the moderate and unregulated epidermal expression of MMP-9 in the hypertrophic and follicular variants of LP. Our findings suggest that the action of MMP-9 is directed toward epidermal/dermal interface, and the cells of inflammatory infiltrates localized subepidermally keep a strong action potential regarding remodeling of the extracellular matrix and basement membrane components. Differences in MMP-9 expression related to collagenous structures clearly appeared in the cases of LS that are characterized by other events underlying the pathogenesis of this dermatosis. The results of the present study suggest that immunologic mechanisms may play a crutial role in the pathogenesis of LP. Our results are in accordance with the data reported by Gunduz [9]. It seems that inflammatory cells are constant producers of 
MMP-9 in LP. Moreover, the application of immunohistochemical technique allowed us to suggest that inflammatory cells are placed in the close vicinity to the vascular beds, which, in turn, are somehow involved in cell migration, and, probably, the further induction of the transformation of the perivascular extracellular matrix. Semiquantitative scoring of MMP-9 expression was helpful in estimation of greatly varying patterns of immunostaining revealed among the cases used in this study. The same approach has been reported in a few the cases published previously [9]. Lowering of MMP-9 expression in the case of LP pemphigoides can be explained by the severe damage of the keratinocytes within the stratum malpighii due to bulla formation, still the inflammatory infiltrate appeared to be heavily stained. Sweat glands constantly showed rather strong MMP-9 expression. In this case explanation can be based on developmental peculiarities when glands and surface epithelium are sharing common characteristics. Still, other explanations may appear from further studies. The analysis of the literature available shows that the enzymatic remodeling caused by MMP-9 has been studied exploring the role of MMPs in the cancerization of oral lichen planus [10]. It has been proved that the risk of cutaneous LP [11] or LS malignizations is very small compared with mucosal involvement [12]. More studies are needed to elucidate the role of MMP-9 in malignancy.

\section{REFERENCES}

1. Pittelkow M. R., Daoud M. S. Lichen planus. In: Wolff GK, Goldsmith L, Katz S, Gilchrest B, Paller A, eds. (2008). Dermatology in general medicine. $7^{\text {th }}$ ed., New York, McGraw-Hill, 244-55.

2. Neill M., Tatnal F. M., Cox N.H. (2002). Guidelines for the management of lichen sclerosus. BJD, 147, 640-649.

3. Wolff K., Johnson R. (2009). Fitzpatrick's Color Atlas and Synopsis of Clinical Dermatology. Sixth Edition, New York, McGraw-Hill Professional, 128-143.

4. Dickie R. J., Horne C. H. W, Sutherland H. W. (1982). Direct evidence of localized immunologic damage in lichen sclerosus et atrophicus. J Clin Pathol, 35, 1395-9.

5. Hewitt J. (1986). Histologic criteria for lichen sclerosus of the vulva. J Reprod Med, 31, 781-7.

6. McKee P. H. (1999). Essential skin pathology. Hong Kong, Mosby International Ltd., 78. 
7. Kulthanan K., Jiamton S., Varothai S., Pinkaew S, Sutthipinittharm P. (2007). Direct immunofluorescence study in patients with lichen planus. International Journal of Dermatology, 46, 1237-1241.

8. Kähäri V. M, Saarialho-Kere U. (1997). Matrix metalloproteinases in skin. Experimental Dermatology, 6, 199-213.

9. Gunduz K., Demireli P., Inanir I., Nese N. (2006) Expression of matrix metalloproteinases (MMP-2, MMP-3, and MMP-9) and fibronectin in lichen planus. J Cutan Pathol, 33, 545-550.

10. Chen Y., Zhang W., Geng N., Tian K., Windsor L. J. (2008). MMPs, TIMP-2, and TGF- $\beta 1$ in the cancerization of oral lichen planus. Head \& Neck, DOI $10.1002 /$ hed, $1237-1245$.

11. Sigurgeirsson B., Lindelof B. (1991). Lichen planus and malignancy: an epidemiologic study of 2071 patients and a review of the literature. Arch Dermatol, 127, 1684.

12. Barbagli G., Palminteri E., Mirri F., Guazzoni G., Turini D., Lazzeri M. (2006). Penile carcinoma in patients with genital lichen sclerosus: a multicenter survey. J Urol, 175(4), 1359-1363.

\section{Address for correspondence:}

Ilze Legusa

Clinical Centre for Skin and Sexually Transmitted Diseases

Maskavas str. 266/9, Riga, LV-1063, Latvia

E-mail: ilze.legusa@inbox.lv 